\title{
Die Qualitätsstrategie des Bundes muss gemeinsam umgesetzt werden
}

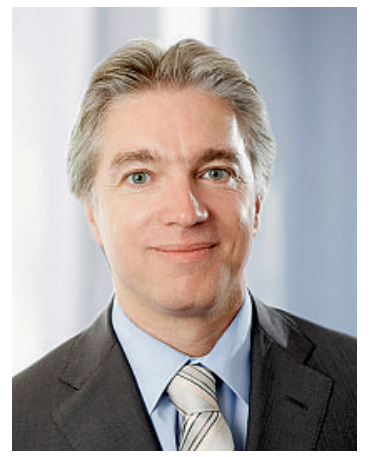

Endlich! Endlich ist es da, das Papier zur Festlegung der Qualitätsstrategie des Bundes. Monatelang wurde es unter Verschluss gehalten und war Objekt von Spekulationen, nun wurde es vom Bundesrat abgesegnet und kann in die Phase der Konkretisierung treten. Was steht denn nun in diesen 121 Seiten? Um es kurz zu machen: Grundsätzlich entspricht das Papier in grossen Zügen unseren Erwartungen und bietet - bei sinnvoller Umsetzung - eine Chance, im Qualitätsbereich in der Schweiz vorwärtszukommen.

Kernstück der Strategie ist ein zu schaffendes nationales Organ, welches die Koordination der Qualitätsaktivitäten im ambulanten wie im stationären Sektor an die Hand nehmen soll. In diesem Sinne beabsichtigt man, die Qualitätsbemühungen in der Schweiz, unter der Führung des Bundes, zu bündeln und zu vernetzen. Der Bund will dabei für eine eindeutige Rollenverteilung sorgen und die Zuständigkeiten zwischen den Akteuren regeln. Die Basis der Strategie bilden neun Aktionsfelder, in welchen, in Anlehnung an die WHO, im Bereich Qualität steuernd eingegriffen werden kann. Das sind Felder wie «Führung und Verantwortung», «Qualitätsinformation», «Anreize» sowie Schaffung von Ressourcen zur Umsetzung. So weit, so gut.

\section{Wir fordern das BAG auf, die Ärzteschaft von An- fang an in die Umsetzung der Qualitätsstrategie einzubeziehen}

Zwei Knackpunkte gibt es aus Sicht der FMH in der Umsetzung dieser Strategie: 1. Ein ausgewogener Einbezug der verschiedenen Akteure in dieser Strategieumsetzung und Konkretisierung ist unabdingbar. Wir fordern vom Bundesamt für Gesundheit (BAG), dass die FMH von Anfang an, zusammen mit den anderen wichtigen Partnern, in die Projekt- umsetzung einbezogen wird. Als Leistungserbringer an der Patientenfront sind es vor allem wir Ärztinnen und Ärzte, die eine sinnvolle und nutzbringende Umsetzung dieser Qualitätsstrategie mitbestimmen müssen. Wir sind bereit, dabei konstruktiv mitzuarbeiten.

\section{Nutzbringende Qualität kostet. Die vorgesehenen 20 bis 25 Millionen Fran- ken sind bescheiden und müssen primär für die Koordination und Einhaltung der gefassten Strategie eingesetzt werden}

2. Die Sicherung und Bereitstellung der Finanzierung ist von entscheidender Bedeutung. Sinnvolle, nutzbringende Qualitätsarbeit kostet. Das Strategiepapier fordert die Bereitstellung von 20 bis 25 Millionen Franken. Je nach Aufgaben dieses nationalen Qualitätsorgans scheint das, angesichts der Gesamtkosten des Gesundheitswesens von über 60 Milliarden, ein bescheidener Betrag zu sein. Damit wird auch klar: Mit einer Finanzierung in dieser Höhe kann bestenfalls eine Plattform zur Verfügung gestellt werden, die sich vor allem auf die Koordination und Strategie konzentriert. Was es aber unbedingt zu verhindern gilt, ist die Schaffung einer zusätzlichen Abteilung im BAG, die isoliert und fern der täglichen Praxis eine Qualitätsstrategie umzusetzen versucht, die niemandem etwas bringt.

Bis die Strategie des Bundes sich konkretisiert und die Vorbereitungsarbeiten abgeschlossen sind, werden die von der FMH geleiteten Qualitätsprojekte - Q-Monitoring, Qualitätsinitiativen und andere (Informationen dazu finden Sie auf www.fmh.ch $\rightarrow$ Weitere Themen $\rightarrow$ Qualität) weitergeführt. Aufgrund der vorliegenden Strategieabsicht des BAG passen sie gut in die medizinische Qualitätslandschaft und können, ungeachtet der späteren nationalen Qualitätsstruktur, integriert werden.

Dr. med. Daniel Herren, MHA, Mitglied des Zentralvorstandes der FMH, Verantwortlicher für das Ressort DDQ 